\title{
Opinion on the recent development of injectable biomaterials for treating myocardial infarction
}

\author{
WANG Wei, LIANG Shuang \& LIU WenGuang* \\ School of Materials Science and Engineering, Tianjin Key Laboratory of Composite and Functional Materials, Tianjin University, \\ Tianjin 300354, China
}

Received April 26, 2017; accepted May 8, 2017; published online June 15, 2017

\begin{abstract}
Citation: Wang W, Liang S, Liu W G. Opinion on the recent development of injectable biomaterials for treating myocardial infarction. Sci China Tech Sci, 2017, 60: 1278-1280, doi: 10.1007/s11431-017-9064-7
\end{abstract}

Myocardial infarction (MI), as one of the most threatening manifestations of cardiovascular disease, remains a therapeutic challenge due to its high morbidity, mortality and recurrence rate [1]. The native myocardial is an electroactive tissue capable of transmitting electrical signal and stimulating an autonomic activity of heart. Typically, MI causes serious damage to the myocardium and leads to adverse scar formation. The disorder of mechanical and electrical signals impairs cardiac function and eventually leads to heart failure. In recent years, diverse technologies have been developed to boost the regeneration of infarcted myocardium, where the injectable biomaterials are increasingly attractive due to minimally invasive surgeries. Additionally, bioactive substances such as stem cells, cytokines and drugs are able to in situ encapsulated in precursor injectable solution and could be conveniently delivered to MI regions.

Natural materials including alginate, chitosan, collagen, fibrin, hyaluronic acid, keratin, and Matrigel have been developed as injectable biomaterials for treating MI. Also, several synthetic materials, such as polyethylene glycol (PEG) and thermoresponsive poly( $N$-isopropylacrylamide) (PNIPAAm), have been employed to construct the injectable biomaterials to treat MI. IK-5001 (1\% sodium alginate plus $0.3 \%$ calcium gluconate) and Algisyl-LVR (a mixture of a $\mathrm{Na}^{+}$-alginate and a $\mathrm{Ca}^{2+}$-alginate) have been used in clinical

*Corresponding author (email: wgliu@tju.edu.cn) trials, which exhibited conservative clinical results [2]. Recently, a hydrogel (VentriGel) derived from decellularized porcine myocardial tissue was shown to exhibit a promising result in treating porcine MI [3]. However, the mechanisms of injectable biomaterials in treating MI are still elusive; they may involve the mechanical support, cell infiltration, and stimulating factors.

The myocardium tissue contracts and relaxes alternately with impulses, and the mechanical signal transmission in the MI area plays a very important role in the repairing process [4]. However, the selection on the modulus of the hydrogel systems from MPa to Pa level is significantly different in various research reports. It is a general belief that hydrogels should possess mechanical properties comparable to that of the native myocardium to provide adequate mechanical support to the infarcted myocardium [5]. However, in our latest research, we found that injectable biomaterials in vitro applications can have a very low stiffness $(25 \mathrm{~Pa})$, and a significant improvement of heart functions (ejection fraction and infarction size) was achieved, which is comparable to the other reports with much stiffer hydrogels. On the other hand, if the biomaterial is too stiff, the mismatched mechanical properties may lead to stress concentration and exert extra loads to the infarcted myocardium. Furthermore, because it would experience full cardiac load along with injection, the injectable biomaterials with excellent fatigue resistant performance is desirable for the application in the dynamic environment [6]. 
Another important consideration is biodegradability, which indicates how quickly the biomaterial could be removed when it was applied in MI. A material must be degraded within a determined time period and the degradation products must be biocompatible, non-toxic, non-immunogenic, and easily metabolized by the host. Pathological remodeling is completed within 6 weeks in a rodent MI, so the scaffold degradation is likely to require a similar time frame. A longer remaining period may hinder repair process. It should be noted that there is still much debate as to the mechanical and degradation properties of injectable materials applied for MI, due to the large disparity between different research reports [2,5].

Synchronized electrical signal transduction is essential for normal myocardial contraction. Therefore, tremendous efforts have been made to fabricate conductive injectable biomaterials for MI treatment. It has been found that the conduction of electrical signals can be improved by hybridizing the conductive carbon nanotubes, metal nanowires, grapheme, and conductive polymers into traditional biomaterials. Conductivity was beneficial for enhancing the interactions among cardiomyocytes (CMs) as well as promoting the maturity of CMs and tissue regeneration [4]. However, the metabolism and biosafety of these conductive particles is still a big concern after being injected into the myocardium.

The injectable biomaterials encapsulating different cytokines applied in vivo could provide biological versatility to improve the cardiac function. It has been realized that systemic inflammation plays a crucial role in the development of MI; thus various anti-inflammatory drugs, such as atorvastatin, rographolide, and resveratrol were employed to down-regulate the pro-inflammation cytokine tumor necrosis factor- $\alpha$ (TNF- $\alpha)$. On the other hand, vascular endothelial growth factor (VEGF), angiopoietin-1 (ANG-1), and angiogenic peptides were introduced into injectable hydrogels with the capacity of promoting angiogenesis. In order to promote stem cell homing, stem cell homing factor (SDF-1) was encapsulated in the hydrogels to induce regeneration of the infarcted myocardium. In recent years, gaseous transmitter (NO, CO, $\mathrm{H}_{2} \mathrm{~S}$ ) and hypoxia inducible factor-1(HIF-1) were explored in cardiovascular disease to promote vascularization as well [7].

A high-level of cell death occurs during MI, so it is necessary to recruit the cardiac progenitor cells to compensate this cell loss. CMs solely contribute to functional contraction with limited ability for in vitro expansion. Stem cells, comprising embryonic stem cells (ESCs), induced pluripotent stem cells (iPSCs), bone marrow mesenchymal stem cells (BM$\mathrm{SCs}$ ), adipose-derived stem cells (ADSCs), cardiac stem cells (CSCs), and endothelial progenitor cells (EPCs), are the optimal sources for myocardial regeneration. In general, the contribution of stem cells to myocardial restoration is believed to work by two independent mechanisms: direct differentiation and paracrine effects. Restoration is mainly mediated by the paracrine effect, whereas direct differentiation only plays a minor role due to the fact that the most of transplanted stem cells would die within a few days in vivo [5]. Analogous to stem cell based therapy, investigations have confirmed the promotional effect of stem cells exosomes (SC-Exos) on tissue repair and regeneration, and it is believed that the exosomes induce the promotion of proliferation and the inhibition of apoptosis. In addition, the utilization of non-self-renewable SC-Exos avoids a lot of safety concerns. Meanwhile, SC-Exos are more easily manufactured, handled, and stored than stem cells themselves. Therefore, SC-Exos show great potential for MI to replace stem cell based therapy [8].

Injectable biomaterials designed to treat MI are frequently administered through intramyocardial and intracoronary routes, or catheter injection. However, all these injection routes are not generally perceived as safe for patients, since they may increase the danger of ventricular rupture. Alternatively, nanoparticles taken by intravenous injection (IV) targeting at MI regions become another innovative approach that would not need surgical procedures performed under general anesthesia, thus avoiding bringing new defects to the weak impaired myocardium [9]. The cardiac tissue possesses abundant vascular system, while MI tends to induce local angiorrhexis which is in favor of aggregating of nanoparticles in infarct area. Studies have shown that the nanoparticles via IV can gather at MI region through enhanced permeability and retention (ERP) effect and thus the loaded drugs or genes can promote the reconstruction of the myocardium function. Recently, a promising report showed that intravenous injection of the matrix metalloproteinase (MMP)-responsive nanoparticles could aggregate into the infarct area within $28 \mathrm{~d}$ [10]. IV treatments minimize potential procedure costs and time, but the biodistribution and clearance of the injected materials remains an important issue and is influenced by the size, shape, and charge of the nanoparticles [9].

In summary, we present the recent developments of injectable biomaterials for treating MI, and propose a number of requisite properties of injectable biomaterials to promote cardiac regeneration. However, as a tissue comprising anisotropic and hierarchical structures, injectable biomaterials with the biomimetic architectures may be favorable for guiding cell function during the healing process. Huge effort has been made by many research groups in developing biomaterials to treat MI, but a standardized approach to measure and compare the functional and morphological properties of the heart after treatment is absent. This may lead to inconsistent results from individual groups. In the near future, the full potential of injectable biomaterials for treating MI would be realized by a collaborative team of materials scientists, biochemists, computational/mechanical engineers, and cardiothoracic surgeons.

This work was supported by the National Natural Science Funds for Distinguished Young Scholar (Grant No. 51325305), the National Natural Science 
Foundation of China (Grant No. 51473117), the National Key Research and Development Program (Grant No. 2016YFC1101301), and Tianjin Municipal Natural Science Foundation (Grant No. 15JCQNJC03300).

1 Benjamin E J, Blaha M J, Chiuve S E, et al. Heart disease and stroke statistics - 2017 update: A report from the american heart association. Circulation, 2017, 135: e146-e603

2 Zhu Y, Matsumura Y, Wagner W R. Ventricular wall biomaterial injection therapy after myocardial infarction: Advances in material design, mechanistic insight and early clinical experiences. Biomaterials, 2017, 129: 37-53

3 Wang R M, Christman K L. Decellularized myocardial matrix hydrogels: In basic research and preclinical studies. Adv Drug Deliv Rev, 2016, 96: 77-82

4 Stoppel W L, Kaplan D L, Black Iii L D. Electrical and mechanical stimulation of cardiac cells and tissue constructs. Adv Drug Deliv Rev, 2016, 96: 135-155

5 Sepantafar M, Maheronnaghsh R, Mohammadi H, et al. Stem cells and injectable hydrogels: Synergistic therapeutics in myocardial re- pair. Biotech Adv, 2016, 34: 362-379

6 Bao R, Tan B, Liang S, et al. A $\pi-\pi$ conjugation-containing soft and conductive injectable polymer hydrogel highly efficiently rebuilds cardiac function after myocardial infarction. Biomaterials, 2017, 122: $63-71$

7 Hnatiuk A P, Ong S G, Olea F D, et al. Allogeneic mesenchymal stromal cells overexpressing mutant human hypoxia-inducible factor $1-\alpha$ (HIF 1- $\alpha)$ in an ovine model of acute myocardial infarction. J Am Heart Assoc, 2016, 5: e003714

8 Luo L, Tang J, Nishi K, et al. Fabrication of synthetic mesenchymal stem cells for the treatment of acute myocardial infarction in micenovelty and significance. Circ Res, 2017, 120: 1768-1775

9 Nguyen M M, Gianneschi N C, Christman K L. Developing injectable nanomaterials to repair the heart. Curr Opin Biotech, 2015, 34: $225-231$

10 Nguyen M M, Carlini A S, Chien M P, et al. Enzyme-responsive nanoparticles for targeted accumulation and prolonged retention in heart tissue after myocardial infarction. Adv Mater, 2015, 27: $5547-5552$ 